\title{
Un site de La Tène au cœur des Mauges : le Pinier à Beaupréau (Maine-et-Loire)
}

A La Tene site in the heart of the Mauges region: Le Pinier, Beaupréau (Maine-etLoire)

\section{Axel Levillayer et Daniel Prigent}

\section{OpenEdition}

\section{Journals}

Édition électronique

URL : https://journals.openedition.org/rao/1327

DOI : $10.4000 /$ rao. 1327

ISSN : 1775-3732

\section{Éditeur}

Presses universitaires de Rennes

\section{Édition imprimée}

Date de publication : 31 décembre 2010

Pagination : 73-84

ISBN : 978-2-7535-1383-9

ISSN : 0767-709X

\section{Référence électronique}

Axel Levillayer et Daniel Prigent, " Un site de La Tène au cœur des Mauges : le Pinier à Beaupréau (Maine-et-Loire) », Revue archéologique de l'Ouest [En ligne], 27 | 2010, mis en ligne le 25 février 2012, consulté le 22 août 2022. URL : http://journals.openedition.org/rao/1327 ; DOI : https://doi.org/ $10.4000 /$ rao. 1327

Ce document a été généré automatiquement le 22 août 2022.

Tous droits réservés 


\title{
Un site de La Tène au cœur des Mauges : le Pinier à Beaupréau (Maine-et-Loire)
}

\author{
A La Tene site in the heart of the Mauges region: Le Pinier, Beaupréau (Maine-et- \\ Loire)
}

Axel Levillayer et Daniel Prigent

1 La commune de Beaupréau se situe au centre des Mauges, région qui occupe le quart sud-ouest du Maine-et-Loire, aux confins de la Vendée et de la Loire-Atlantique (fig. 1). Ses alentours sont riches d'un patrimoine archéologique protohistorique et antique, organisé notamment autour des mines d'or de Saint-Pierre-Montlimart (Levillayer, 2003). Un dense réseau d'établissements ruraux (Fricot et al., 2006) se structure autour du site protohistorique et antique de la Ségourie au Fief-Sauvin ${ }^{1}$ (Bouvet et Levillayer 2010 ; Chéné 1982).

2 En 1974, Claude Lambert, à l'occasion d'une campagne de prospection aérienne, photographie un double enclos quadrangulaire au lieu-dit Le Pinier (fig. 2). Cinq années plus tard, un projet de lotissement à cet emplacement amène Daniel Prigent, du Service archéologique départemental de Maine-et-Loire alors nouvellement créé, à intervenir. La fouille, particulièrement difficile, s'est déroulée dans l'urgence alors que les travaux de terrassement avaient déjà débuté.

3 Pour diverses raisons, seuls quelques rares éléments du site ont jusqu'alors été étudiés et publiés (Aubin, 1983 ; Lejars, 2007). Le site apparaissait alors relativement isolé de par sa chronologie ancienne. Dans le cadre d'une reprise des sites protohistoriques fouillés par le Service archéologique départemental, nous présentons ici les résultats complets de cette opération, qui prend d'autant plus de valeur qu'elle peut désormais être mise en perspective avec d'autres sites fouillés depuis lors dans la région. 


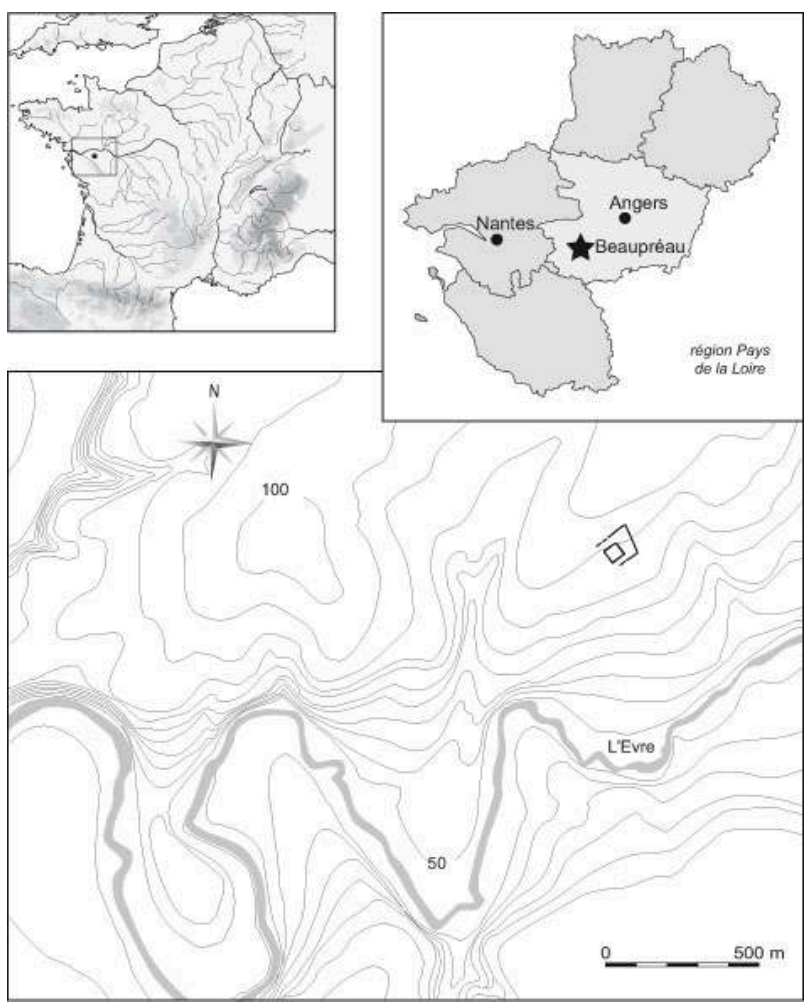

Figure 1 : Localisation du site du Pinier à Beaupréau.

Figure 1: Location of Le Pinier at Beaupréau.

\section{La conduite de l'opération}

4 La photographie aérienne laisse apparaître deux enclos emboîtés (fig. 2). L'enclos interne, dont on perçoit la taille importante des fossés, est discernable dans sa totalité ; il s'agit d'un enclos quadrangulaire. L'enclos externe en revanche n'apparaît que dans sa partie nord, délimité par un fossé rectiligne. Une interruption du fossé pourrait exister dans sa partie ouest (fig. 3).

Le but de la fouille était de vérifier le plan du site, d'en proposer une datation et d'en déterminer la fonction. Par manque de temps, l'ensemble des fossés n'a pas été fouillé ; des sondages ponctuels ont été réalisés en différents points de ceux-ci et au centre de l'enclos. Treize d'entre eux ont été réalisés à la pelle mécanique dotée d'un godet lisse et terminés à la main. Seul le sondage 10, dans l'angle sud-ouest de l'enclos interne, a été mené intégralement à la main, permettant d'en dresser la coupe stratigraphique et de récolter un mobilier plus abondant qu'ailleurs. 


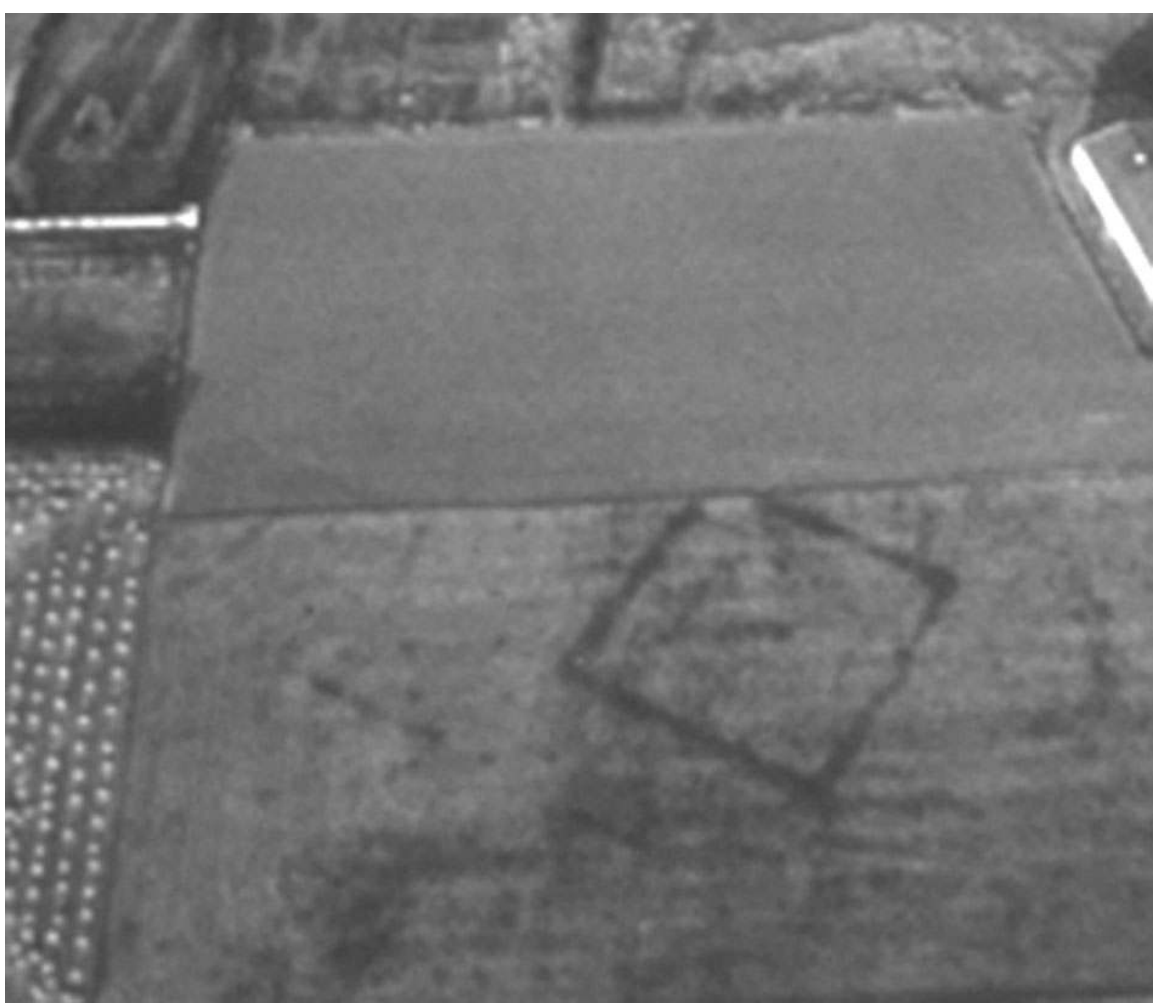

Figure 2 : Le site du Pinier lors de sa découverte en prospection aérienne (cl. : C. Lambert).

Figure 2: Le Pinier as revealed by air survey.

6 Seuls trois sondages et une coupe ont pu être réalisés dans l'enclos externe, la poursuite des travaux de terrassement ayant empêché de poursuivre plus avant son examen. Il n'est donc pas possible de déterminer le tracé exact de cette enceinte, même si les observations faites sur le terrain peuvent être complétées par celles de la photographie aérienne. Les recherches se sont donc concentrées sur l'enclos interne, complétées par un sondage de taille restreinte réalisé à l'intérieur de celui-ci.

\section{Les structures découvertes}

7 Le site du Pinier est installé en position topographique dominante, sur le versant nord de la vallée de l'Evre, à $500 \mathrm{~m}$ de cette rivière qui décrit de nombreux méandres, et à une altitude moyenne de $85 \mathrm{~m}$ NGF (fig. 1). Les terrains sous-jacents sont constitués de schistes briovériens qu'entaille profondément l'Erve, créent un relief marqué.

La fouille a permis de confirmer le plan de l'enclos interne mais pas d'observer l'intégralité de l'enclos externe (fig. 3). Quant au sondage central, il a révélé de très importantes perturbations dues aux labours qui sont probablement à l'origine de la destruction des structures en creux qui ont pu exister.

\section{L'enclos interne}

9 Cet enclos adopte un plan grossièrement carré dont les côtés mesurent environ $40 \mathrm{~m}$. Le fossé a des dimensions relativement imposantes, notamment au regard de ceux d'autres sites du second âge du Fer fouillés dans les Mauges (Fricot et al., 2006). À son niveau d'apparition (à 0,3 m environ sous le sol actuel), il mesure en moyenne 3,50 m à 
l'ouverture, ses dimensions étant comprises entre 3,10 $\mathrm{m}$ du côté est et 3,95 $\mathrm{m}$ du côté nord. Sa profondeur, de l'ordre de $2 \mathrm{~m}$ dans la partie nord et ouest, atteint $2,60 \mathrm{~m}$ au sud. Il présente un profil en $\mathrm{V}$ dissymétrique à fond arrondi (fig. 4). La face externe est toujours plus régulière et présente toujours une déclivité plus prononcée que la face interne.

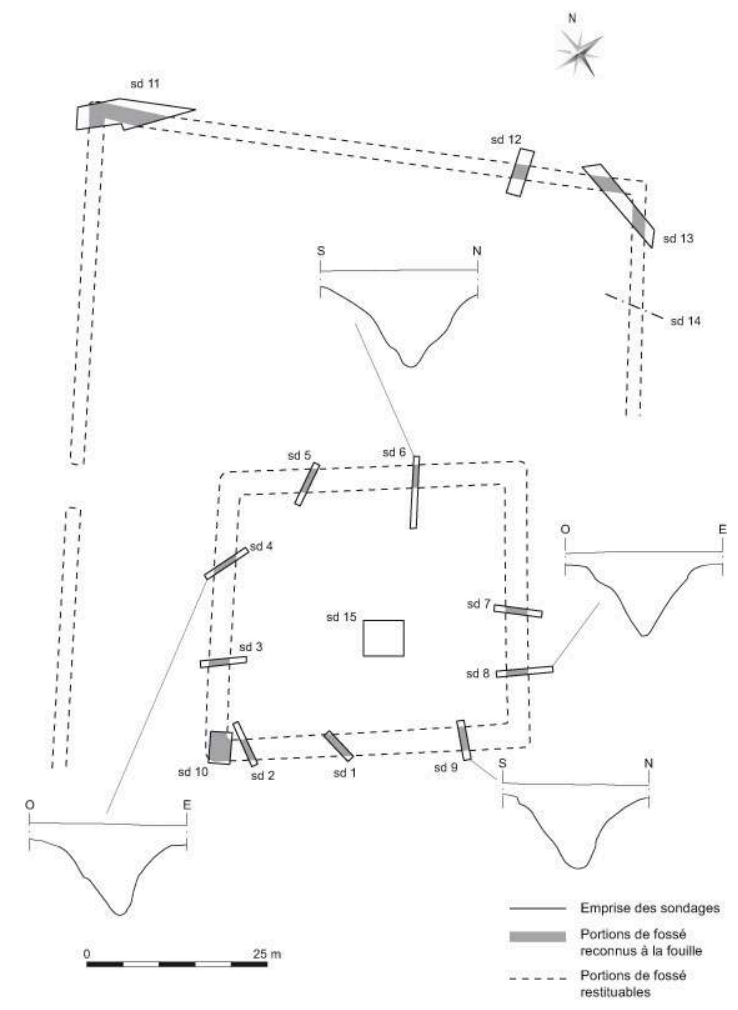

Figure 3 : Plan du site avec quelques coupes significatives du fossé interne.

Figure 3: Plan of the site and some critical sections of the inner enclosure ditch.

La coupe stratigraphique relevée sur le sondage 10 montre des dépôts de limons le long des parois, qui tendent à prouver que le fossé a fonctionné ouvert. La succession des couches qui se sont déposées de façon relativement homogène, en cuvette et sur des épaisseurs parfois considérables, incite à envisager que ce fossé est resté ouvert pendant toute la durée d'occupation du site (Menez, 1996, p. 26 et 30). Des pierres, distribuées de manière asymétrique dans le comblement, accréditent la présence éventuelle d'un talus (l'existence d'un parement, suggérée par une morphologie systématiquement assez plane des pierres, est davantage sujette à caution). 


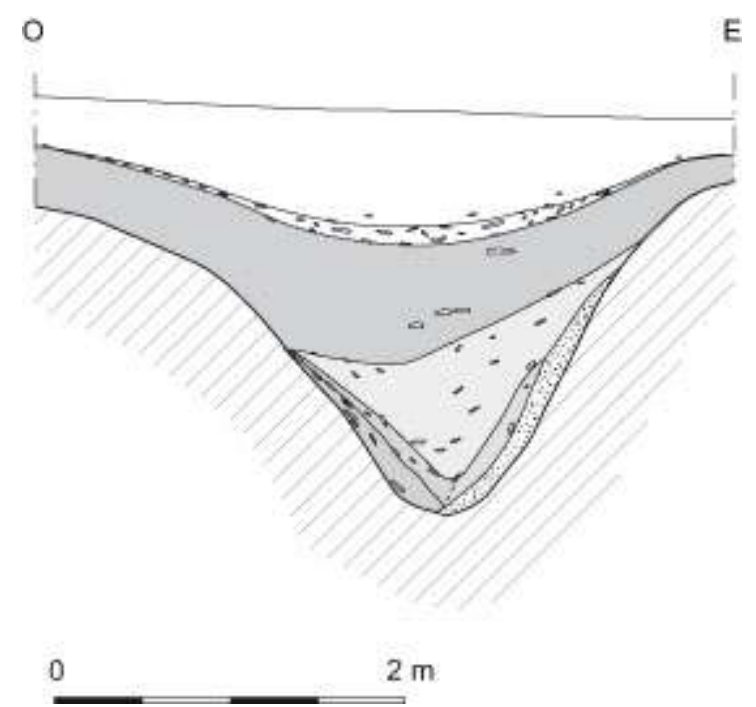

Figure 4 : Coupe stratigraphique du fossé d'enclos interne (sondage 10).

Figure 4: Ditch stratigraphy of inner enclosure (from trench 10).

\section{L'enclos extérieur}

Le fossé de l'enclos externe (au plan légèrement trapézoïdal) a des dimensions plus modestes (fig. 5). Il mesure environ 1,70 m d'ouverture dans sa partie basse, mais un creusement en terrasse dans la partie ouest l'agrandit à $2,55 \mathrm{~m}$ sous la surface. Le profil en $\mathrm{V}$ est très évasé, avec un fond presque plat. Le flanc ouest montre plusieurs décrochements, dont un d'importance qui pourrait correspondre à un recreusement. La stratigraphie relevée dans le sondage 11 irait en ce sens; en effet, les plus anciennes couches correspondent, à cet endroit, à des colluvionnements. Un niveau limoneux et pierreux, qui semble s'être déposé en cuvette, est recoupé par une couche qui vient rejoindre le probable curage ou recreusement évoqué précédemment. Il est donc possible qu'il y ait eu deux phases de fonctionnement. Cependant, le caractère limité des sondages n'a pas permis de les identifier clairement, ni d'interpréter davantage.

\section{Le mobilier}

L'étude du mobilier de l'âge du Fer, majoritairement constitué par de la céramique et présentant des éléments remarquables, est d'autant plus intéressante qu'elle peut être aujourd'hui replacée dans son contexte régional grâce aux fouilles de sites contemporains, intervenues entre-temps aussi bien dans le Maine-et-Loire qu'en LoireAtlantique. Elle permet également de préciser les réflexions engagées à partir des rares éléments du site jusqu'alors publiés (Lejars, 2007 ; Levillayer, 2003).

\section{La céramique}

\section{La céramique indigène}

13 Le nombre des restes s'élève à 686 pour un minimum de 55 individus (calculé à partir des seuls bords) et un poids total de $11,30 \mathrm{~kg}$. Ce lot est donc modeste mais non dénué d'intérêt. 
14 Du fait des conditions de fouille, le plan de répartition du mobilier n'a pas de réelle signification. Tout au plus, on note que la très grande majorité du mobilier provient de l'enclos interne (511 tessons et 47 individus). Le sondage 10, effectué manuellement, concentre à lui seul 284 tessons pour un NMI de 23.

Une typologie formelle, complétée par des observations sur le montage des vases, a été élaborée de façon empirique ; quinze types ont été définis (fig. 6 et 7).

D'un point de vue technique, seules deux grandes catégories ont été distinguées: la céramique modelée d'une part et la céramique tournée ou régularisée au tour lent d'autre part. La première catégorie est majoritaire et représente $78 \%$ des productions présentes sur le site. On classe parmi les $22 \%$ restants les céramiques tournées sur lesquelles des traces de tour rapide ont pu être identifiées, ainsi que les céramiques qui ont été finies à la tournette.

17 Les pâtes sont globalement homogènes. La majorité peut se ranger dans la catégorie des pâtes communes sombres, la cuisson étant, sauf exceptions, réductrice (le mode réducteur primitif dans lequel la privation d'oxygène est incomplète, est très majoritaire : on ne compte que quinze cuissons totalement réductrices). Seuls quelques vases (11 au total), peuvent être qualifiés de pâtes claires ; parmi ceux-ci, quatre sont du type 11a (fig. 7).

18 Les vases grossiers, essentiellement modelés, se répartissent préférentiellement sur quelques types. Ce sont les formes 9,10 et 12, mais aussi les jattes à panse semihémisphérique de type 3 (fig. 6, 7). Les céramiques fines sont exclusivement tournées. Elles ne concernent que les types $4 \mathrm{~b}$ et $11 \mathrm{~b}$, ainsi que les formes remarquables que nous étudions ci-dessous.

Le dégraissant est généralement quartzeux. L'utilisation de chamotte est cependant à signaler dans trois cas. Enfin, quatre vases, tous de types différents, présentent une pâte micacée qui résulte de l'emploi d'une matrice argileuse vraisemblablement différente des argiles utilisées pour les autres céramiques. 


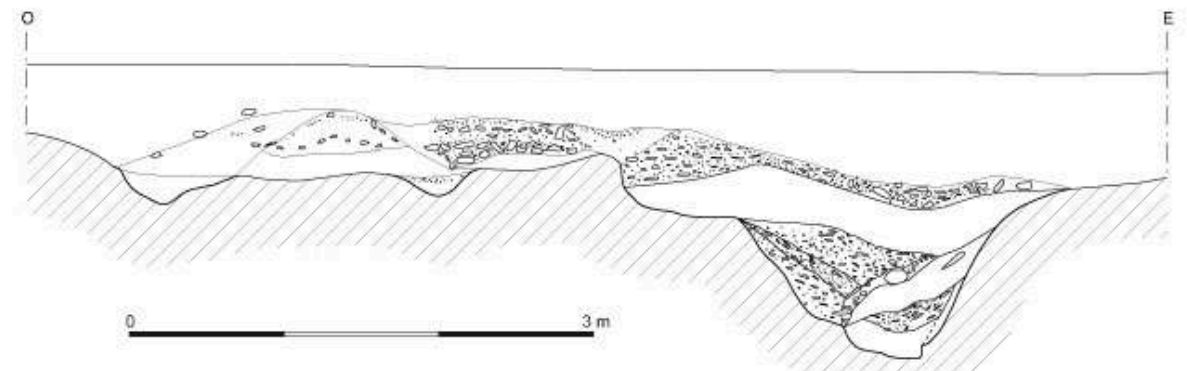

Figure 5 : Coupe stratigraphique du fossé d'enclos extérieur (sondage 11). Figure 5: Ditch stratigraphy of outer enclosure (from trench 17).

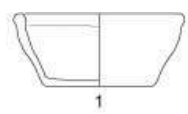

0
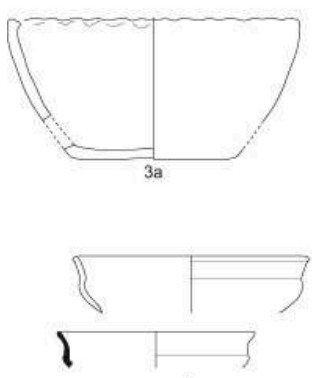

$4 a$

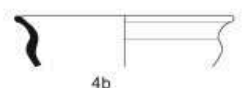

2a
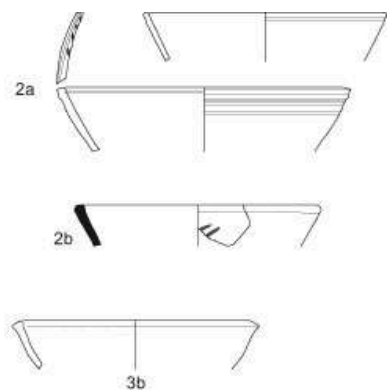
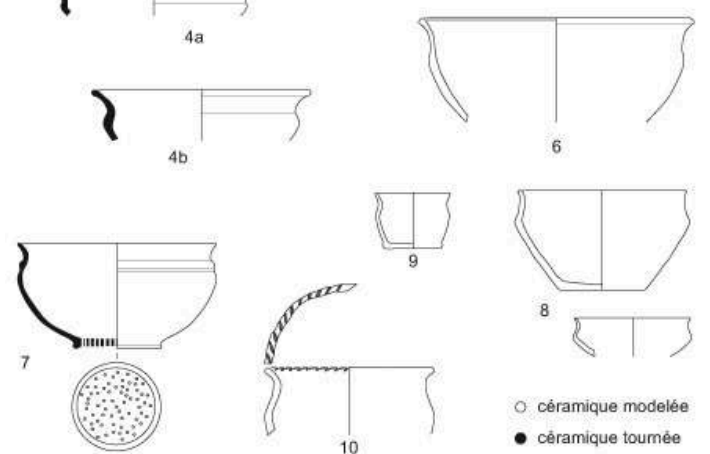

Figure 6: Typologie céramique de l'enclos interne. Figure 6: Typology of pottery from the inner enclosure. 

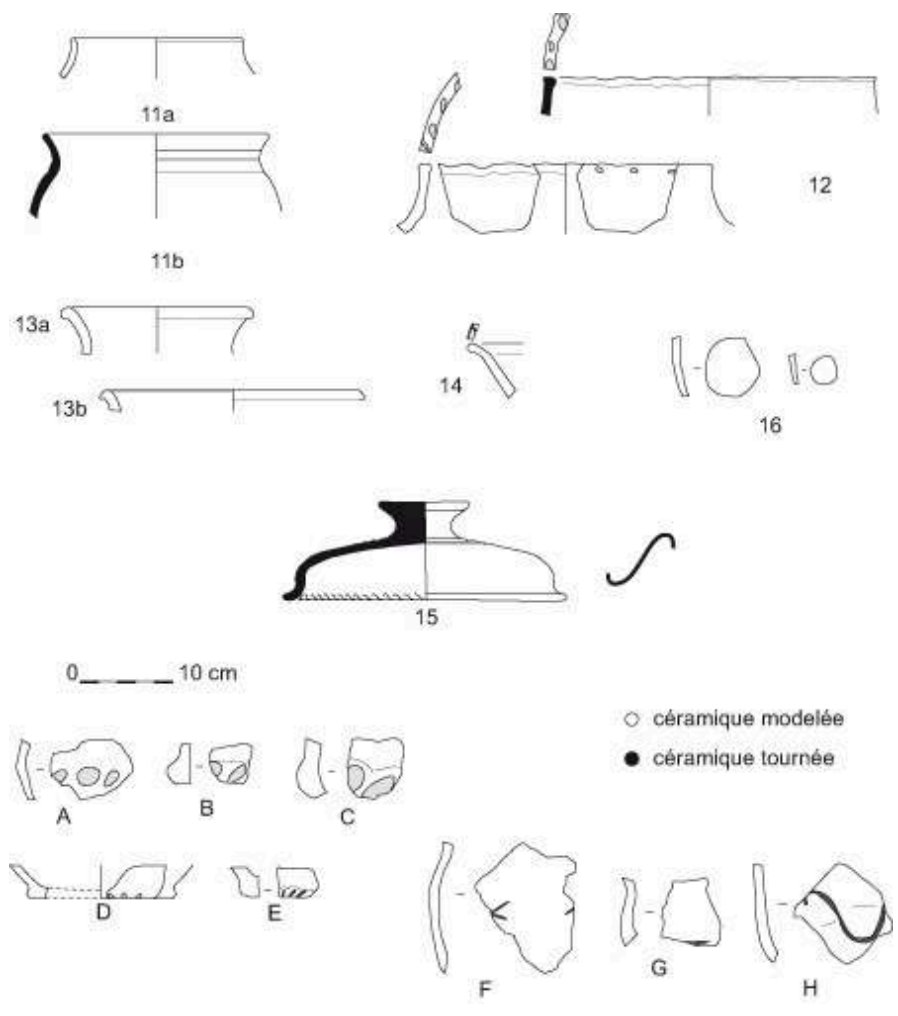

Figure 7 : Typologie céramique et faciès décoratif de l'enclos interne. Figure 7: Typology and decoration of pottery from the inner enclosure.

\section{Présentation des types}

Notre type 3 est constitué de jattes modelées, grossières, à panse semi-hémisphérique et lèvre pseudo-rentrante (celle-ci pouvant être ornée de légers festons ou de digitations) qui trouvent dans la région plusieurs éléments de comparaison : on en rencontre sur les 
établissements de Marcé (Nillesse, 2003, p. 159-160), ainsi que dans la phase 2 des Chaloignes à Mozé-sur-Louet (Levillayer, 2006, p. 124). Sur les sites mentionnés, ces vases sont montés au tour et datés de La Tène $\mathrm{C} 1$ et $\mathrm{C} 2$. Ils paraissent plus soignés que ceux du Pinier qui évoquent, notamment par leurs décors, des productions plus anciennes.

Les écuelles carénées à hauts cols déjetés constituent le type 4. Ces formes sont assez répandues en Gaule du nord et de l'ouest pour les périodes anciennes et moyennes de La Tène. Le sous-type $4 a$ trouve un bon équivalent dans la Vienne, à Civeaux «la Papotière » (Gomez de Soto et al., 2007, p. 77). Plus près de Beaupréau, c'est encore une fois la première phase du site de la Reculière qui fournit les meilleurs parallèles, avec des exemplaires plus décorés toutefois. Le sous-type $4 b$ quant à lui semble plus tardif.

Le type 5 correspond à un individu unique. Il s'agit d'une céramique fine, tournée, qui présente une cannelure labiale interne large de $0,18 \mathrm{~cm}$. Ce type de lèvre arrondie éversée rappelle des contextes bretons, comme ceux du Boisanne datés de La Tène $C$ (Menez, 1996, p. 110-112).

Une jatte à profil en $S$ évasé avec une lèvre très légèrement en bourrelet (type 6) appelle des comparaisons avec des contextes régionaux datés plutôt de La Tène $C$, aux Chaloignes (Levillayer, 2006) ou encore sur le site du Moulin des Courtes aux Moutiersen-Retz en Loire-Atlantique (Levillayer en préparation).

Le type 7 a été créé pour définir la passoire découverte dans le sondage 10. Ce vase se distingue du reste du corpus à la fois et par sa typologie et par son mode de fabrication. Cette écuelle en $\mathrm{S}$ au profil relativement globulaire présente un petit pied annulaire percé avant la cuisson. Elle est ornée d'un cordon et d'une cannelure sur la panse. La pâte est fine. Le vase a été monté au tour rapide et cuit en mode réducteur total, achevant de souligner l'aspect soigné du récipient.

De manière générale, les passoires ne se multiplient dans les contextes d'habitat qu'à partir de la fin de La Tène moyenne (Adam, 2002). Auparavant, dans l'Ouest, ils restent rares. Dans le Maine-et-Loire, on en connaît un exemplaire orné tout à fait exceptionnel à Brion (Barbier, 1995). D'un point de vue formel, ce vase évoque les jattes moyennes à cordons connues en Bretagne pour la fin de l'âge du Fer (Daire, 1992, pl. 12). Ici toutefois, le pied et la lèvre évoquent des contextes plus anciens (première moitié de La Tène moyenne ?). Il est donc difficile de dater précisément ce vase qui ne trouve pas d'exact équivalent et on doit se contenter d'envisager La Tène moyenne ou finale.

29 Les jattes moyennes du type 8 sont modelées; elles présentent une carène relativement vive et un point d'inflexion relativement haut dégageant un col individualisé. Elles se rapprochent à nouveau de récipients découverts à la Reculière, mais aussi de formes connues dans tout le quart sud-ouest du Maine-et-Loire, entre La Tène $\mathrm{C}$ et La Tène $\mathrm{D}$.

Un seul vase trapu caréné à col concave constitue le type 9, peu caractéristique.

31 Les pots de type 10, à profil en $\mathrm{S}$ adouci et lèvre profondément digitée montrent une pâte grossière cuite en mode réducteur primitif; ils se rencontrent à Brion (dans des variantes plus anguleuses toutefois) et à Saint-Philbert-de-Grandlieu en LoireAtlantique (Bellanger en préparation).

32 Le type 11, avec dix-huit vases, est le mieux représenté de notre corpus. Il regroupe l'ensemble des pots ovoïdes à col plus ou moins éversé simple. Certains ont même des cols sub-verticaux. La distinction que nous y avons établie repose sur la finesse de la 
pâte et sa mise en œuvre. Le sous-type 11a définit les vases modelés dont un tiers présente en surface des teintes très claires. Les pots en pâte fine montés au tour constituent le sous-type $11 b$ (cinq individus); ils sont parfois ornés de fines cannelures sur le col. Ce sous-type est attribuable à une phase avancée du second âge du Fer centrée sur le ii ${ }^{\mathrm{e}}$ siécle av. n. è. et évoque à nouveau les contextes de l'ouest de la Gaule. Tout comme pour les précédents, les vases de type 12 ne sont connus que par leurs parties hautes. Ils se distinguent du type précédent par leur lèvre sub-verticale à bourrelet interne et à décor digité sommital. Ce type de lèvre nous semble tout à fait caractéristique des ensembles angevins de La Tène ancienne, sans qu'on puisse exclure de le retrouver dans le reste de la région.

Les types 13 et 14 ne sont documentés que par leurs hauts cols, respectivement concaves et tronconiques.

Le type 15 correspond au seul vase jusqu'alors publié. La pâte est fine, presque "savonneuse ». Le vase a été monté au tour rapide. Il est orné d'une frise de esses estampés, ainsi que de cannelures et a été cuit, tout comme la passoire, en mode réducteur total. Il a ensuite été lustré sur sa surface externe.

On peut hésiter quant à l'identification de ce vase: coupe ou couvercle? D'abord interprété comme une coupe (Aubin 1983), il a ensuite été considéré comme un couvercle. Cet objet est très proche d'un couvercle découvert à Plouër-sur-Rance (Menez, 1996, p. 118), et encore davantage d'un exemplaire retrouvé dans le souterrain de l'habitat de Prat-Kergourognon, également dans les Côtes d'Armor (Milcent, 1993, pl. 79). Le premier est daté de la seconde moitié du iiie siècle, alors que la datation du second oscille entre les iii et iie siècles av. n. è. Récemment cependant, Thierry Lejars a proposé d'identifier le vase du Pinier à une coupe à pied (Lejars, 2007, p. 274), en la rapprochant d'une coupe haute à pied découverte dans le sanctuaire de Mirebeau (Côte-d'Or), voire de la passoire de Brion, et en arguant du fait que les couvercles présentent habituellement un pied creusé. En cela, elle s'inspirerait de modèles méditerranéens.

Les exemples cités pourraient cependant relever de typologies différentes: le vase de Mirebeau présente un profil d'écuelle plus profonde et la passoire de Brion, plus proche de notre exemplaire, est dotée d'une anse. À l'inverse, les comparaisons avec les contextes bretons et son profil évasé nous semblent devoir rapprocher le vase de Beaupréau d'un couvercle, même si la largeur du « pied » laisse en effet planer le doute.

Enfin, notons la présence de deux jetons en céramique.

Sans qu'on puisse les rattacher à des formes précises, plusieurs décors ont été identifiés. Les digitations, limitées aux céramiques grossières, peuvent orner la panse (fig. 7, A) ou le pied (D, E). Deux cordons digités ont été retrouvés (B, C). Ce type de décor évoque des périodes anciennes; on le trouve au premier âge du Fer et, dans des proportions moindres, aux $v^{e}$ et iv ${ }^{e}$ siècles av. $n$. è.

Outre deux décors incisés (fig. 7, F, G), un décor à la limite de l'incision et du lustrage, ornant vraisemblablement le diamètre maximal de la panse d'un vase grossier, a été appliqué à l'aide d'une petite spatule, (fig. 7, H) ; il représente une esse étirée. Ce motif curviligne est connu dans le Berry à La Tène ancienne (Augier, et al., 2007, p. 140).

41 À cause des conditions de fouille, le fossé extérieur n'a livré que peu de mobilier, et seulement trois individus céramiques (fig. 8). Il s'agit d'une écuelle carénée à haut col sub-vertical qu'on peut rattacher au type 4, et de deux pots ovoïdes à cols courts 
également sub-verticaux mesurant 18 et $22 \mathrm{~cm}$ de diamètre à l'ouverture. Ces derniers se distinguent des formes hautes découvertes dans le fossé de l'enclos interne.
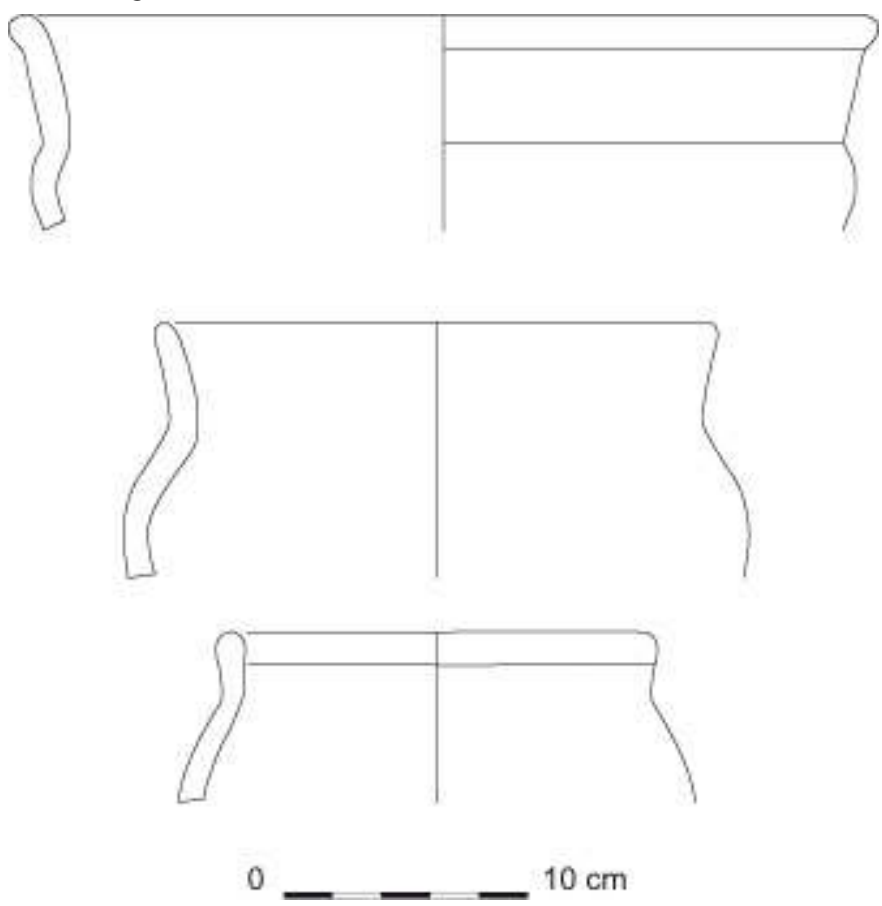

Figure 8: Céramiques découvertes dans le comblement du fossé extérieur.

Figure 8: Pottery from the fill of the outer ditch.

\section{Les amphores}

La découverte de cinq fragments d'amphores républicaines dans le secteur de l'enclos externe atteste de la fréquentation du site au moins jusqu'au début du iie siècle av. $n$. è. Il s'agit de fragments de panses d'un poids total de $0,68 \mathrm{~kg}$ et dont l'attribution typologique est impossible.

\section{Les terres cuites diverses}

Le fossé de l'enclos interne a livré une grande quantité de torchis. En tout, ce sont 202 restes qui ont été dénombrés, pour un poids total de $4,50 \mathrm{~kg}$. Ces fragments attestent de constructions à l'intérieur de l'enclos central. Le sondage 10 a également livré des restes de plaques foyères (18 au total). Elles mesurent entre 2 et 3,5 $\mathrm{cm}$ d'épaisseur avec une moyenne se situant à $3 \mathrm{~cm}$. La majorité de ces plaques foyères ne présente pas de caractéristiques particulières à l'exception de l'une d'entre elles à rebord arrondi. Enfin, on note la présence de six fragments de boudins en argile. Ceux-ci ont été soumis à des températures importantes, en une ou plusieurs fois. Ces éléments moulés à la main évoquent les boudins de calage connus dans les fours à sel et destinés à y bloquer les récipients à saumure. On peut penser qu'ils aient servi ici dans des activités de cuisson d'autres matériaux. 


\section{Le métal}

\section{La fibule} comparaison sont tous extra-régionaux. En effet, à l'exception de la fibule à décor plastique de Juvigné en Mayenne, aucune autre fibule de La Tène ancienne n'a été découverte dans la région. Il s'agit ici d'une fibule en bronze à corde interne et probablement à deux fois deux spires (fig. 9). Son arc filiforme mouluré permet de la rattacher à La Tène B (Lejars, 2007, p. 273). Elle évoque une parenté certaine avec des fibules de type « Dux », par exemple celles du type I (Kruta, 1973, p. 22).

Ces fibules à arc étiré en seraient des modèles dérivés tardifs qui annoncent les fibules de La Tène moyenne. On trouve ainsi des formes proches dans la phase de transition définie par Waldhauser entre La Tène B1a et La Tène B1b-1c et jusqu'à La Tène B2a (Waldhauser, 1987, fig. 4). Une comparaison peut être établie avec la fibule découverte au Châtelard à Rivières (Charente), datable de la fin de La Tène ancienne ou du tout début de La Tène moyenne (Kerouanton, 2009, p. 118).
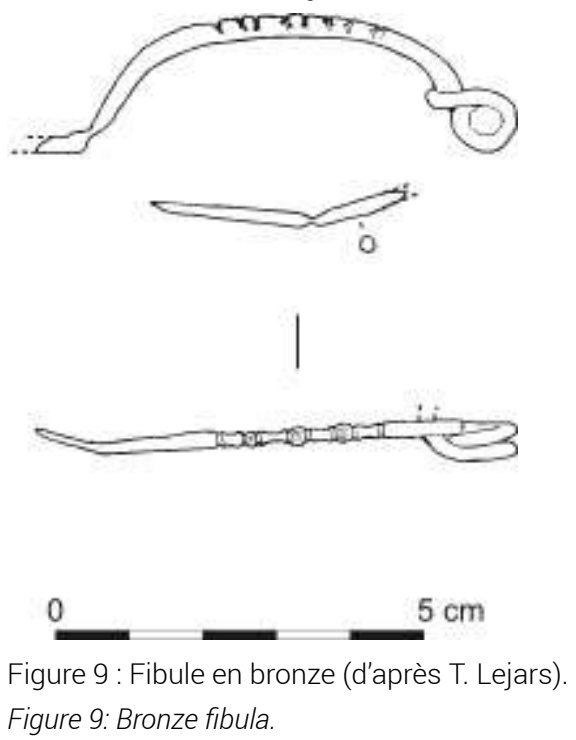

Le fer

Des fragments de fer très corrodés ont aussi été découverts. Il s'agit de deux fragments légèrement concaves retrouvés dans le sondage 10 et de trois fragments découverts dans le sondage 14 (enclos extérieur). Ajoutons à cela la présence de scories de fer mises au jour dans le fossé de l'enclos interne.

\section{Le lithique}

Le fossé d'enclos externe a livré un fragment de meule rotative en granite, ainsi qu'un probable peson en schiste perforé (fig. 10). Le fossé d'enclos interne a pour sa part livré un broyon en granite.

Dix-neuf éclats de silex, dont un certain nombre retouchés, ont également été découverts dans le fossé interne. Ils proviennent des mêmes couches que la céramique et ne doivent plus être considérés comme des artefacts intrusifs. En effet, l'usage d'un 
outillage lithique est avéré à l'âge du Fer, et plus particulièrement sur les sites artisanaux (Humphrey, 2007).

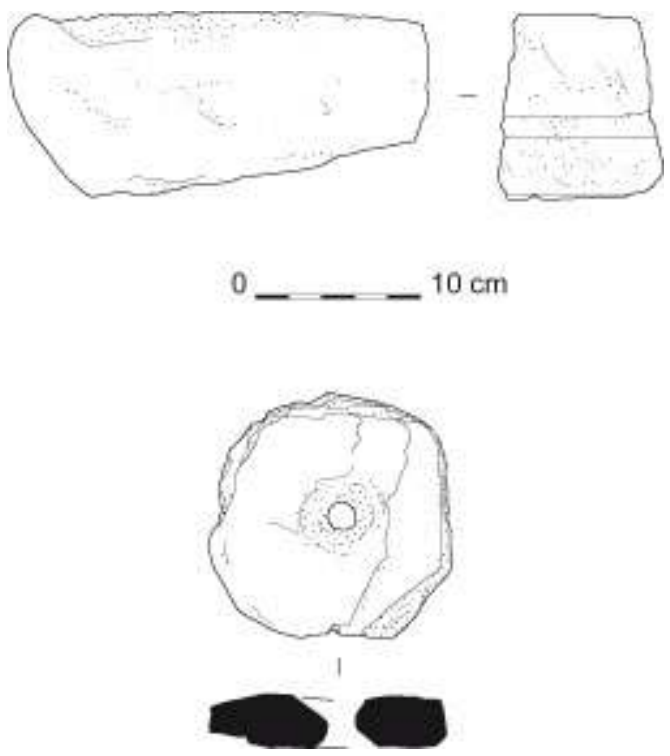

Figure 10 : Mobilier lithique du fossé extérieur.

Figure 10: Lithic artefacts from the outer ditch.

\section{Datation de l'ensemble céramique et fonctionnement des enclos : un site occupé de la fin de La Tène ancienne à la fin de La Tène moyenne?}

L'étude du mobilier issu de l'enclos interne montre une certaine hétérogénéité de la céramique. Un certain nombre d'éléments renvoient à une phase ancienne qu'on peut attribuer à un horizon compris entre le milieu du iv ${ }^{e}$ siècle et le iiie siècle. La fibule participe de cet horizon puisqu'elle peut être attribuée à La Tène B1 ou B2.

Le mobilier céramique montre des affinités notables avec les sites de la Gravouillerie à Saint-Philbert-de-Grandlieu (Loire-Atlantique) et surtout avec la première phase d'occupation de la Reculière à Ecouflant, datée de La Tène $\mathrm{C} 1$, où ont été découverts des éléments d'armement de La Tène B2 (Nillesse, 2004).

51 À Beaupréau, cette première phase de fonctionnement du site pourrait être plus précisément attribuée à une phase finale de La Tène B2, ou plutôt à une phase de transition La Tène B2-C1 observée de plus en plus fréquemment en Gaule (voir par exemple: Gomez de Soto et al., 2007, p. 77). Cette phase, correspondant à la fin du iv ${ }^{\mathrm{e}}$ siècle av. $n$. è. et au début du siècle suivant, est illustrée par exemple par certains types céramiques et en particulier par les jattes à panse semi-hémisphérique qui annoncent les formes de La Tène $\mathrm{C}$. Le « couvercle » estampé doit également être attribué à cette phase, de même que les vases de stockage de type 12, ou encore les décors de cordons digités et de digitations.

La découverte d'éléments plus tardifs dans le fossé interne, en particulier des vases tournés des types $4 \mathrm{~b}, 5$ et $11 \mathrm{~b}$, semble attester d'une occupation qui perdure au moins jusqu'au iie siècle av. $n$. è. Bien que le mobilier découvert y soit rare, la plus grande partie du comblement du fossé externe pourrait relever de cette période centrée sur la 
fin de La Tène moyenne ou le début de La Tène finale (La Tène C2, voire D1a), comme l'atteste la découverte de quelques fragments d'amphores républicaines ou encore celle d'un élément de meule rotative, forcément postérieure au iiie siècle av. n. è.

Il n'est toutefois pas impossible, comme le suggère la stratigraphie, que ce fossé ait connu un premier état de fonctionnement correspondant peut-être à l'occupation la plus ancienne du site. Les deux fossés auraient pu fonctionner de concert pendant toute la durée de fonctionnement du site.

Le Pinier est donc occupé sur au moins deux siècles, entre le iv ${ }^{\mathrm{e}}$ et le ii ${ }^{\mathrm{e}}$ siècle av. n. è. Corrélé à celui d'autres sites, dont ceux d'Ecouflant, le mobilier ancien permet d'entrevoir un faciès angevin de la fin de La Tène ancienne et du début de La Tène moyenne dominé par les jattes tronconiques (parfois à bord légèrement infléchi) et les écuelles carénées à haut col déjeté, faciès qui s'intègre parfaitement à ceux connus dans le reste de la Gaule du nord à la même époque. La présence d'un décor estampé est un élément intéressant. En effet, depuis plusieurs années, les exemples de décors estampés se multiplient dans les Pays de la Loire, en particulier dans le Maine-et-Loire et en Mayenne, ainsi que dans le Poitou (Gomez de Soto, 2006 ; Levillayer en préparation). Ces découvertes attestent d'une dynamique artistique propre à cet espace nordoccidental atlantique au cours de La Tène ancienne et moyenne, depuis la Bretagne jusqu'au Poitou et aux marches de la Normandie (Gomez de Soto, 2006).

\section{Interprétation du site}

55 L'étude du plan du site et de son mobilier amène à s'interroger quant à sa nature. En effet, les enclos multiples emboîtés et concentriques ont pendant longtemps été perçus comme des sanctuaires. C'est une théorie envisagée notamment par Thierry Lejars en s'appuyant sur le plan quadrangulaire de l'enclos interne qui évoque les sanctuaires connus de la fin de La Tène ancienne et de La Tène moyenne, tels que celui de Gournaysur-Aronde (Lejars, 2007). Notons qu'un double enclos de plan parfaitement centré a également été découvert en photographie aérienne au lieu-dit Marsillé sur la commune de Beaupréau par Gilles Leroux (INRAP).

56 Au Pinier, la présence d'une passoire soignée et d'une " coupe-couvercle », qui serait alors à identifier comme une coupe à boire, pourrait évoquer le domaine du vin et, dans ces chronologies hautes, celui du banquet. Cette même sphère rituelle était évoquée sur le site de la Reculière (Nillesse, 2004) dont on a vu les affinités qu'il entretient avec le Pinier. Cependant, ici, la contemporanéité de la passoire et de la "coupe-couvercle » n'est pas assurée. Enfin, les jetons en céramique sont parfois envisagés comme intervenant dans des pratiques rituelles, peut-être liées au vin ou à la boisson (Viand, 2008, p. 58-59). Toutefois, on trouve de tels jetons - et en grand nombre - sur l'habitat ouvert groupé des Pichelots aux Alleuds (Gruet et Passini, 1986) où aucune pratique rituelle ne peut être décelée.

57 L'identification du site du Pinier comme un sanctuaire se heurte à d'autres objections. Tout d'abord son plan centré n'est pas certain, d'une part parce que la fouille du deuxième enclos n'en a pas touché la partie sud, et d'autre part parce que nous ne sommes pas assurés de l'exacte contemporanéité des deux fossés, ni qu'il faille systématiquement associer ces plans à des sanctuaires. À Azé (Mayenne), au lieu-dit la Mazure, Claude Lambert et Jean Rioufreyt ont fouillé une triple enceinte quadrangulaire à plan parfaitement centré, repérée d'avion et alors envisagée comme 
une enceinte cultuelle. L'étude spatialisée du mobilier a montré que ce plan est probablement le résultat de remaniements (et certainement d'agrandissements), entre la fin de l'âge du Fer et la période gallo-romaine, d'un habitat caractérisé aussi bien par la céramique que par l'instrumentum Il semble qu'il en soit de même au Pinier où l'étude de l'ensemble du mobilier tend à l'assimiler à un habitat du fait de la présence de mobilier lithique (dont des éléments de mouture), de nombreuses plaques foyères et de boudins à probable usage artisanal.

qui y a été retrouvé (Levillayer en préparation).

Cette interprétation n'est toutefois pas forcément antinomique par rapport à d'éventuelles pratiques rituelles qui, ici, ne sont pas forcément à chercher dans le plan du site. En effet, pour les périodes hautes de l'âge du Fer, les sphères profanes et sacrées pourraient s'associer dans un même espace, pourquoi pas celui d'une certaine élite, celle-là même qui consomme le vin. Et puis, à La Tène moyenne et finale, on sait l'existence de gestes non domestiques sur les habitats (Bouvet et al., 2003 ; Levillayer, 2006)

60 Si l'hypothèse d'un habitat semble la plus pertinente, il n'en reste pas moins qu'il a livré un certain nombre de mobiliers peu fréquents, parmi lesquels le "couvercle » à décor estampé et la fibule en bronze qui, toutes proportions gardées eu égard à la modestie des sondages, posent la question du statut social des habitants du site.

61 L'importance du fossé de l'enclos interne pourrait venir appuyer l'idée d'une population relativement aisée. Cependant, ces dimensions ne doivent pas être surinterprétées car elles restent bien inférieures à celles des enclos des habitats que l'on peut véritablement qualifier d'aristocratiques comme celui de Paule, ou celui des Natteries à Cholet dont la profondeur atteint 3,40 m pour une largeur maximale de $8 \mathrm{~m}$ (Maguer, 2007, p. 80). Ici, manquent également les marqueurs caractéristiques de cette aristocratie, tel l'armement (Menez, 2008).

Alors qui étaient les habitants du Pinier? La statue au torque découverte à moins d'un kilomètre du site, et peut-être contemporaine de son occupation (Olivier, 2003), perpétuerait-elle le souvenir de l'un d'entre eux? Ce serait alors un argument supplémentaire en faveur de l'importance du site et de son secteur à l'âge du Fer, dont une des possibles explications est la richesse du sous-sol des Mauges, région dont le site du Pinier vient éclairer la connaissance des mobiliers, à une période charnière du Second âge du Fer qui reste en grande partie à documenter dans la région.

Nous remercions Thierry Lejars (ENS - UMR 8546) pour ses conseils et ses réflexions avisés, ainsi que les relecteurs du manuscrits pour leurs conseils.

\section{BIBLIOGRAPHIE}

Adam, A.-M., 2002 - Les passoires dans le monde celtique : formes, origine, usage, in Meniel, P. et Lambot, B. (coord.), Découvertes récentes de l'âge du Fer dans le massif des Ardennes et ses marges. Repas des vivants et nourriture pour les morts en Gaule (actes du XXXVe colloque 
international de l'AFEAF, Charlevilles-Mézières, 2001). Société archéologique champenoise (Mémoire $n^{\circ}$ 16, suppl. au Bulletin nº 1), p. 143-156.

Aubin, G., 1983 - Informations archéologiques, Circoncription des Pays de la Loire, Gallia, 41 (fasc. 2), p. 312.

Augier, L., Mennessier-Jouannet, C., Milcent, P.-Y., Orengo, L. et Riquier, S., 2007 - La France centrale aux iv ${ }^{\mathrm{e}}$-iiie siècle av. n. è. (Auvergne, Berry, Orléanais), in Mennessier-Jouannet, C., Adam, A.-M. et Milcent, P.-Y. (dir.), La Gaule dans son contexte européen aux IVe et IIIe siècles avant notre ère (actes du XXVII ${ }^{\mathrm{e}}$ colloque international de l'AFEAF, Clermont-Ferrand, 2003). Lattes, ADALR (Monographies d'Archéologie méditerranéennes), p. 117-176.

Barbier, S., 1995 - Brion. La Croix-Boizard, (DFS de fouille de sauvetage urgent), Nantes, SRA des Pays de la Loire, 2 vol.

Bellanger, P., en préparation - Le site de la Galonnière à Saint-Philbert-de-Grandlieu (LoireAtlantique).

Bouvet, J.-P. et Levillayer, A. (avec la coll. de Sicard, S., 2010 - Les Celtes et l'essor du monde rural, in Morin, E. (coord.), Emergence. Archéologie et histoire du Choletais (catalogue d'exposition), Cholet, Ville de cholet, p. 39-50.

Chêne, J., 1982 - Segora, l'antique mansion romaine enfin révélée par le cadastre du Fief-Sauvin, Beaupréau, (compte d'auteur), $94 \mathrm{p}$.

Daire, M.-Y., 1992 - Les céramiques armoricaines de la fin de l'âge du Fer. Rennes, Université de Rennes 1 (Travaux du Laboratoire d'Anthropologie, 39), 316 p.

Fricot, L., Crowch, A., Hunot, J.-Y. et Montaudon, M., 2006 - Aménagement de la R.D. nº 752 entre Beaupréau et Saint-Léger-sous-Cholet. Construction d'échangeurs et mise en $2 \mathrm{X} 2$ voies. Maine-et-Loire. Andrezé, Bégrolles-en-Mauges, Saint-Macaire-en-Mauges, Saint-Léger-sousCholet (rapport de diagnostic d'archéologie préventive), Nantes, SRA des Pays de la Loire, 124 p.

Gomez de Soto, J., 2006 - L'art laténien du v et du iv siècles av. J.-C. en Gaule de l'Ouest : monde nord-alpin et/ou méditerranéen? Actualités de l'art celtique d'Occident, in Frère, D. (dir., avec la coll. de Morin, A.), De la Méditerranée vers l'Atlantique. Aspects des relations entre la Méditerranée et la Gaule centrale et occidentale (viIIe-IIe siècles av. J.-C.), Rennes, PUR, p. 57-65.

Gomez de Soto, J., Lejars, T., Duconge, S., Robin, K., Sireix, C. et Zelie, B., 2007 - Du milieu $d u v^{e}$ au iii ${ }^{e}$ s. av. n. è. en Centre-Ouest, Aquitaine septentrionale et ouest du massif Central, in Mennessier-Jouannet, C., Adam, A.-M. et Milcent, P.-Y. (éd.), La Gaule dans son contexte européen aux IVe et IIIe siècles avant notre ère (actes du XXVII ${ }^{\mathrm{e}}$ colloque international de l'AFEAF, Clermont-Ferrand, 2003). Lattes, ADALR (Monographies d'Archéologie méditerranéennes), p. 69-89.

Gruet, M. et Passini, B., 1986 - Le village ouvert des Pichelots, Dossiers Histoire et Archéologie, $n^{\circ} 106$, p. $18-23$.

Humphrey, J., 2007 - Simple tools for thought tasks or thought tools for simple tasks ? Analysis and experiment in Iron Age flint utilisation, in Haselgrove, C. and Pope, R. (ed.), The Earlier Iron Age in Britain and the near Continent, Oxford, Oxbow Books, p. 144-159.

Kerouanton, I., 2009 - Rivières, Le Châtelard (Charente). Un site de hauteur fortifié protohistorique. Fouilles programmées 2000-2005. Premiers éléments, in Bertrand, I., Duval, A., Gomez de Soto, J. et Maguer, P. (dir.), Les Gaulois entre Loire et Dordogne (actes du XXXI colloque international de l'AFEAF, Chauvigny 2007). Chauvigny, APC (Mémoire XXXIV), p. 113-119. 
Kruta, V., 1973 - Remarques sur les fibules de la trouvaille de Duchcov (Dux), Bohême, in Duval, P.-M. (dir.), Recherches d'Archéologie celtique et gallo-romaine, Hautes Études du monde grécoromain, 5, p. 21-36.

Lejars, T., 2007 - Lieux de culte et pratiques votives en Gaule à La Tène ancienne, in MennessierJouannet, C., Adam, A.-M. et Milcent, P.-Y. (éd.), La Gaule dans son contexte européen aux IVe et IIIe siècles avant notre ère (actes du XXVII colloque international de l'AFEAF, Clermont-Ferrand, 2003). Lattes, ADALR (Monographies d'Archéologie méditerranéenne), p. 265-282.

Levillayer, A., 2003 - Les Mauges (Maine-et-Loire) : archéologie d'un terroir du premier âge du Fer au début du haut Moyen-Âge (viIIe av.-VIIIe s. apr. J.-C.). Occupation du sol et peuplement (mémoire de maîtrise d'archéologie), Rennes, Université de Rennes 2, 2 vol.

Levillayer, A., 2006 - L'occupation de l'âge du Fer aux Chaloignes (Mozé-sur-Louet, Maine-etLoire), Revue archéologique de l'Ouest, 23, p. 117-135.

Levillayer, A., en préparation - Territoire, chronologie et société : faciès céramiques et horizons chrono-culturels autour de la basse vallée de la Loire (vie-Ier s. av. n. è.) (thèse d'archéologie, Université de Rennes 1).

Maguer, P., 2007 - Le site aristocratique des Natteries, Le Puy-Saint-Bonnet (Maine-et-Loire), in Bertrand, I., et Maguer, P. (dir.), De pierre et de terre. Les Gaulois entre Loire et Dordogne (catalogue d'exposition), Chauvigny, Association des publications chauvinoises (Mémoire XXX), p. 78-80.

Menez, Y., 1996 - Une ferme de l'Armorique gauloise : Le Boisanne à Plouër-sur-Rance (Côtesd'Armor), Paris, éditions de la Maison des Sciences de l'Homme (DAf 58), 267 p.

Menez, Y., 2008 - Le camp de Saint-Symphorien à Paule (Côtes-d'Armor) et les résidences de l'aristocratie du second âge du Fer en France septentrionale (thèse de doctorat), Paris, université de Paris 1 (Panthéon-Sorbonne), 2 vol.

Milcent, P.-Y., 1993 - L’Âge du Fer en Armorique à travers les ensembles funéraires (ix ${ }^{\mathrm{e}}$ iiie siècles avant J.-C.), Antiquités nationales, 25, p. 17-50.

Nillesse, 0., 2003 - Les établissements ruraux gaulois de Marcé (Maine-et-Loire) et la hiérarchisation des oppida, in Mandy, B. et de Saulce, A. (dir.), Les Marges de l'Armorique à l'âge du Fer. Archéologie et Histoire ; culture matérielle et sources écrites (actes du XXIII ${ }^{\mathrm{e}}$ colloque de l'AFEAF, Nantes, 1999), Revue archéologique de l'Ouest, suppl. 10, p. 149-172.

Nillesse, 0., 2004 - Le fourreau d'épée à décor d'appliques de la Reculière (Maine-et-Loire) et son contexte, Antiquités nationales, 36, p. 215-228.

Olivier, L., 2003 - La Dame de Beaupréau (Maine-et-Loire) : une nouvelle statue gauloise au torque du iie s. av. J.-C., Antiquités nationales, 35, p. 13-17.

Viand, A. (dir.), 2008 - Nanterre et les Parisii. Une capitale au temps des Gaulois ?, (catalogue d'exposition), Paris, Somogy, $128 \mathrm{p}$.

Waldhauser, J., 1987 - Keltische Gräberfelder in Böhmen, Bericht RGK, 68, p. 25-179. 


\section{NOTES}

1. Une notice détaillée recensant les très nombreuses découvertes faites sur le site a été rédigée dans le cadre du PCR Agglomérations secondaires antiques de Bretagne et Pays de la Loire par G. Aubin, J.-P. Bouvet, A. Levillayer, M. Monteil et M. Mortreau.

\section{RÉSUMÉS}

Découvert à l'occasion de prospections aériennes, le site du Pinier a été le premier dans la région à livrer un ensemble mobilier dont une partie date du IV siècle av. n. è. Le site, occupé pendant au moins deux siècles, se présente sous la forme d'un double enclos à plan rectilinéaire avec fossés massifs. On ne sait pas si les deux enclos sont contemporains ou si ce plan résulte d'une évolution dans le temps. Si sa fonction reste à confirmer (habitat ou sanctuaire ?), on y trouve en tout cas un mobilier riche et jusqu'alors peu fréquent dans les contextes régionaux contemporains (fibule, passoire, céramique décorée...).

Discovered by aerial survey, the site of le Pinier was the first in the region to produce an assemblage partly dating from the 4th Century BC. The site, occupied at least for at least two centuries, is in part a double rectilinear enclosure with large ditches. The two enclosures cannot be separated by sequence or time. Although function is not certain (settlement or sanctuary?), a rich locally exceptional assemblage (fibula, ceramic strainer, decorated ceramic...) was found.

\section{INDEX}

Mots-clés : La Tène ancienne, Maine-et-Loire, céramique, fibule, sanctuaire, habitat, enclos Keywords : Early La Tene, Maine-et-Loire, ceramics, fibula, sanctuary, settlement, quadrangular enclosure

\section{AUTEURS}

\section{AXEL LEVILLAYER}

Service archéologique départemental de Maine-et-Loire, doctorant Université de Rennes 1, UMR 6566 CReAAH. (a.levillayer@cg49.fr)

\section{DANIEL PRIGENT}

Service archéologique départemental de Maine-et-Loire, UMR 5594 ARTeHIS (d.prigent@cg49.fr) 